\title{
Unil
}

UNIL | Université de Lausanne

Unicentre

$\mathrm{CH}-1015$ Lausanne

http://serval.unil.ch

Year : 2013

\section{Inexperienced Sonographers Can Successfully Visualize and Assess a Three-Dimensional Image of the Fetal Face Using a Standardized Ultrasound Protocol}

\author{
Guenot Cecile
}

Guenot Cecile, Inexperienced Sonographers Can Successfully Visualize and Assess a Three-Dimensional Image of the Fetal Face Using a Standardized Ultrasound Protocol, 2013

Originally published at : Thesis, University of Lausanne

Posted at the University of Lausanne Open Archive http://serval.unil.ch

Document URN : urn:nbn:ch:serval-BIB_1D23E478A91E2

\section{Droits d'auteur}

L'Université de Lausanne attire expressément l'attention des utilisateurs sur le fait que tous les documents publiés dans l'Archive SERVAL sont protégés par le droit d'auteur, conformément à la loi fédérale sur le droit d'auteur et les droits voisins (LDA). A ce titre, il est indispensable d'obtenir le consentement préalable de l'auteur et/ou de l'éditeur avant toute utilisation d'une oeuvre ou d'une partie d'une oeuvre ne relevant pas d'une utilisation à des fins personnelles au sens de la LDA (art. 19, al. 1 lettre a). A défaut, tout contrevenant s'expose aux sanctions prévues par cette loi. Nous déclinons toute responsabilité en la matière.

\section{Copyright}

The University of Lausanne expressly draws the attention of users to the fact that all documents published in the SERVAL Archive are protected by copyright in accordance with federal law on copyright and similar rights (LDA). Accordingly it is indispensable to obtain prior consent from the author and/or publisher before any use of a work or part of a work for purposes other than personal use within the meaning of LDA (art. 19, para. 1 letter a). Failure to do so will expose offenders to the sanctions laid down by this law. We accept no liability in this respect. 
UNIVERSITE DE LAUSANNE - FACULTE DE BIOLOGIE ET DE MEDECINE

Département de Gynécologie et Obstétrique

Inexperienced Sonographers Can Successfully Visualize and Assess a Three-Dimensional Image of the Fetal Face Using a Standardized Ultrasound Protocol

\section{THESE}

préparée sous la direction du Docteur Yvan Vial

et présentée à la Faculté de biologie et de médecine de I'Université de Lausanne pour l'obtention du grade de

DOCTEUR EN MEDECINE

par

Cécile GUENOT

Médecin diplômé de la Confédération Suisse

Originaire du Landeron (Neuchatel)

Lausanne

2013 
UNIL | Université de Lausanne

Faculté de biologie et de médecine

Ecole Doctorale

Doctorat en médecine

\section{Imprimatur}

Vu le rapport présenté par le jury d'examen, composé de

Directeur de thèse Monsieurle Docteur Yvan Vial

Co-Directeur de thèse

Expert Monsieur le Professeur Jean-Yves Meuwly

Directrice de l'Ecole Madame le Professeur Stephanie Clarke

doctorale

la Commission MD de l'Ecole doctorale autorise l'impression de la thèse de

\section{Madame Cécile Guenot}

intitulée

Inexperienced Sonographers Can Successfully Visualize and Assess a Three-Dimensional Image of the Fetal Face Using a Standardized Ultrasound Protocol

Lausanne, le 26 novembre 2013

pour Le Doyen

de la Faculté de Biologie et de Médecine

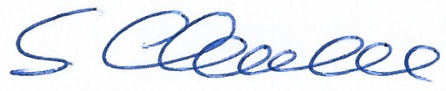

Madame le Professeur Stephanie Clarke Directrice de l'Ecole doctorale 
Fetal Diagnosis and Therapy
Received: November 25, 2012

Accepted after revision: April 2, 2013 Published online: June 18, 2013

\title{
Inexperienced Sonographers Can Successfully Visualize and Assess a Three-Dimensional Image of the Fetal Face Using a Standardized Ultrasound Protocol
}

\author{
C. Guenot D. Baud K. Lepigeon K. Francini M.-C. Rossier P. Hohlfeld Y. Vial
}

Departments of Gynecology and Obstetrics, University Hospital of Lausanne CHUV, Lausanne, Switzerland

\section{Key Words}

Cleft lip · Fetal face $\cdot 3 \mathrm{D}$ ultrasound $\cdot$ Post-processing ·

Prenatal diagnosis $\cdot$ General screening $\cdot$ Profile .

Learning curve

\begin{abstract}
Introduction: A standardized three-dimensional ultrasonographic (3DUS) protocol is described that allows fetal face reconstruction. Ability to identify cleft lip with 3DUS using this protocol was assessed by operators with minimal 3DUS experience. Material and Methods: 260 stored volumes of fetal face were analyzed using a standardized protocol by operators with different levels of competence in 3DUS. The outcomes studied were: (1) the performance of post-processing 3D face volumes for the detection of facial clefts; (2) the ability of a resident with minimal 3DUS experience to reconstruct the acquired facial volumes, and (3) the time needed to reconstruct each plane to allow proper diagnosis of a cleft. Results: The three orthogonal planes of the fetal face (axial, sagittal and coronal) were adequately reconstructed with similar performance when acquired by a maternal-fetal medicine specialist or by residents with minimal experience (72 vs. $76 \%, p=0.629$ ). The learning curve for manipulation of 3DUS volumes of the fetal face corresponds to 30 cases and is independent of the operator's level of experience. Discussion: The learning curve for the standardized protocol we de-
\end{abstract}

scribe is short, even for inexperienced sonographers. This technique might decrease the length of anatomy ultrasounds and improve the ability to visualize fetal face anomalies.

Copyright $\odot 2013$ S. Karger AG, Basel

\section{Introduction}

Congenital fetal malformations affect approximately $2.5-3 \%$ of all live births and are an important cause of perinatal morbidity and mortality [1-3]. Cleft lips and palates are among the most common nonlethal congenital malformations with a prevalence ranging from 1 to 2.69 per 1,000 births depending on ethnicity $[4,5]$. Nevertheless, prenatal detection of these malformations remains low in the general population, especially when clefts are isolated $[1,6]$. Only $18 \%$ of isolated cleft lips are identified, whereas the detection increases to $44 \%$ when associated with other anomalies [1]. However, large variations have been observed between and within countries ( $0-75 \%$; $50 \%$ in our county).

In the local EUROCAT (Lausanne), the detection rate of fetal facial clefts over the last 10 years was 32\% [1].

Prenatal ultrasound examination for fetal facial anomalies has long been recommended and routine screening is a standard of care in many countries. Prenatal identifi- 
Fig. 1. Two-step study analyzing 260 fetal facial volumes acquired between 17 and 38 weeks of gestational age.

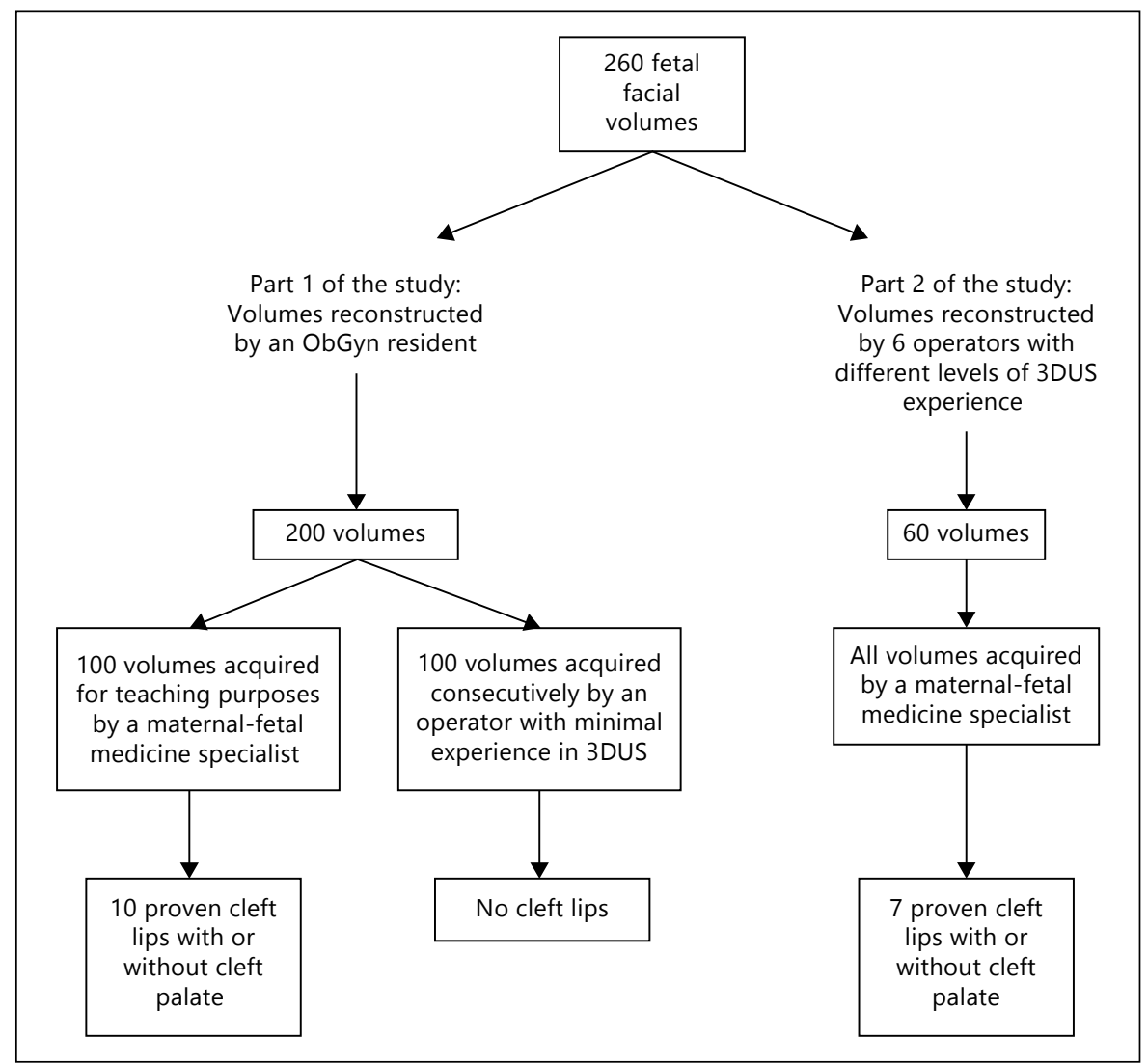

cation of such malformations allows adequate counseling of the parents, psychological preparation for birth and anticipation for future surgery with a multidisciplinary pediatric team [7]. Moreover, the presence of a cleft lip is associated with more than 100 syndromes with a lethal or significantly disabling prognosis.

In our department, conventional two-dimensional ultrasonography (2DUS) is used to screen for malformations. Introduction of three-dimensional ultrasonography (3DUS) in the 1990s has added more information in the analysis of abnormal images obtained in 2DUS.

To obtain adequate $2 \mathrm{D}$ images of the fetal face can be challenging and time-consuming. Fetal facial assessment is often a reason for referral to third-level centers which can lead to unnecessary stress for the patient.

To facilitate fetal facial analysis and improve visualization of cleft lips, we conducted a study to assess the feasibility of 3DUS to alleviate the technical difficulties in obtaining the different planes necessary to analyze the fetal face. We also aimed to assess the efficacy of 3DUS in the visualization of cleft lips when used by operators with minimal 3DUS experience.

\section{Materials and Methods}

The goal of this study was (1) to describe a standardized protocol to analyze the fetal face using 3DUS and (2) to assess the role of 3 DUS to detect fetal facial anomalies in general prenatal screening.

We performed a two-step study and analyzed a total of $260 \mathrm{fe}$ tal facial volumes acquired between 17 and 38 weeks of gestational age (fig. 1). These volumes were acquired during examinations performed for early morphologic scan or intrauterine growth control.

All volumes were obtained with a Voluson E8, a 730 Expert or a 730 Pro (GE Healthcare, Zipf, Austria) and were blindly manipulated under the supervision of a maternal-fetal medicine specialist.

We retrospectively reviewed all stored volumes of fetal faces from the unit's database that were acquired from January 1 to December 31, 2008. Of note, the operator with minimal experience in 3DUS acquired 100 3DUS volumes consecutively (fig. 1). The maternal-fetal medicine specialist acquired 100 nonconsecutive volumes for teaching purposes. In order to obtain high-quality 3DUS volumes of the fetal face, we recommend to wait for the absence of active fetal movements and maternal breathing movements, to exercise minimal pressure on the patient abdomen to increase amniotic fluid amount in front of the face, to bring the 3DUS probe as sagittal as possible compared to the fetal head $( \pm 10 \%)$ using a $30^{\circ}$ insonation angle, and the fastest acquisition possible. 
Table 1. Protocol for obtaining the three orthogonal plane images of the fetal face

1 The image is magnified to increase the structural details (three-quarters of the fetal face filling the image)

2 Contrast and threshold are adjusted to eliminate shadows or echoes and details refined by changing the gain

3 The cursor dot is set on the tip of the nose in the three orthogonal plane images

4 In the axial plane, the nose is set at the 12-o'clock position and the level of the orbits is balanced until they appear symmetric

5 In the sagittal plane, the tip of the nose, the upper lip and the chin are rotated until they are in line with the horizontal axis

6 In the sagittal plane, the cursor dot is set on the upper lip, facing the maxilla

7 The acquired image displays:

a The axial plane at the level of the upper lip and maxilla

b A strict profile

c The coronal plane with the tip of the nose, the upper lip, the lower lip and the chin

8 The palate can be analyzed if the cursor dot is moved from the front of the face to the occiput on the coronal plane

All operators of this study attended a 1-hour teaching session on the basic principle of $\mathrm{X}-\mathrm{Y}-\mathrm{Z}$ rotation and on evaluation of the three orthogonal planes (axial, sagittal and coronal). The standardized three orthogonal plane images of the face were obtained using the technique summarized in table 1 and the $\mathrm{Z}$ rotation as described by Abuhamad et al. [8].

In the first part of the study, a resident with 2DUS experience but whose only 3DUS experience was attending the teaching session mentioned above attempted to reconstruct 100 volumes acquired by a maternal-fetal medicine specialist. Of note, obstetrics and gynecology ultrasounds are not performed by radiologists in our country, but by obstetricians and gynecologists, and each resident has at least a 6-month ultrasound rotation. These volumes included 10 proven cleft lips with or without cleft palate. The resident then attempted to reconstruct another 100 volumes obtained by an operator with minimal experience in 3DUS volume acquisition (resident in obstetrics and gynecology learning routine anatomy ultrasounds in low-risk pregnancies).

In the second part of the study, we evaluated the learning curve of 6 operators with different levels of 3DUS experience. Among the 6 operators, 1 is a maternal-fetal medicine specialist, 2 are fellows in fetal medicine, 1 is a resident in obstetrics and gynecology (learning routine anatomy ultrasounds in low-risk pregnancies) and 2 are sonographers with more than 8 years' experience (performing routine anatomy ultrasounds in low-risk pregnancies). A total of $603 \mathrm{DUS}$ volumes of the fetal face acquired by a maternal-fetal medicine specialist were used. Seven volumes with cleft lips with or without cleft palates were randomly mixed with normal volumes. We analyzed the time required to reconstruct the best orthogonal planes.

When fetal face volumes were analyzed, we tested the ability of the different operators to reconstruct the three orthogonal plane images: (1) axial plane: view of the upper lip and maxilla (fig. 2a), (2) sagittal plane: view of the profile (fig. $2 \mathrm{~b}$ ), and (3) coronal plane: section crossing the tip of the nose, the upper lip, the lower lip and the tip of the chin (fig. 2c). The reconstruction of the fetal face was defined as accurate when these three plane images were adequately visualized.

All 17 clefts included in this study were unilateral, involving the palate in 10 cases. No median clefts were included.

3DUS Fetal Face Assessment by

Inexperienced Sonographers
Data were reviewed either directly on the Voluson or on a laptop workstation with the 4D View program (GE Healthcare), which allows manipulation of the volumes. The volumes were displayed in multiplanar images.

All data were gathered and statistical analyzes were performed using Stata 11.0 (StataCorp, College Station, Tex., USA). Multilevel mixed-effects linear regression models were used to analyze the learning curves.

The study was approved by the Institutional Review Board of the University of Lausanne, Switzerland.

\section{Results}

\section{Ability of an Inexperienced Ultrasound Operator to Reconstruct Adequate Fetal Facial Planes}

I. Analysis of the Volumes Acquired by the

Maternal-Fetal Medicine Specialist

The 100 volumes used in this component of the study were acquired from a sagittal view. Among them, two were isolated cleft lips and eight also involved the maxilla. All ten clefts were correctly recognized by the resident. Of note, all defects concerning the hard palate, when present, were also recognized by the resident.

Analysis of the three orthogonal plane images resulted in the correct identification of the upper lip in the axial plane in $91 \%$ of cases, of the maxilla in the axial plane in $84 \%$ of cases, of the fetal profile in $92 \%$ of cases, and of the upper lip in the coronal plane in 95\% of cases (table 2). Adequate reconstructions of all facial components from the same volume (upper lip in a coronal plane, maxillary in an axial plane and facial profile) were possible in $72 \%$ of cases. 


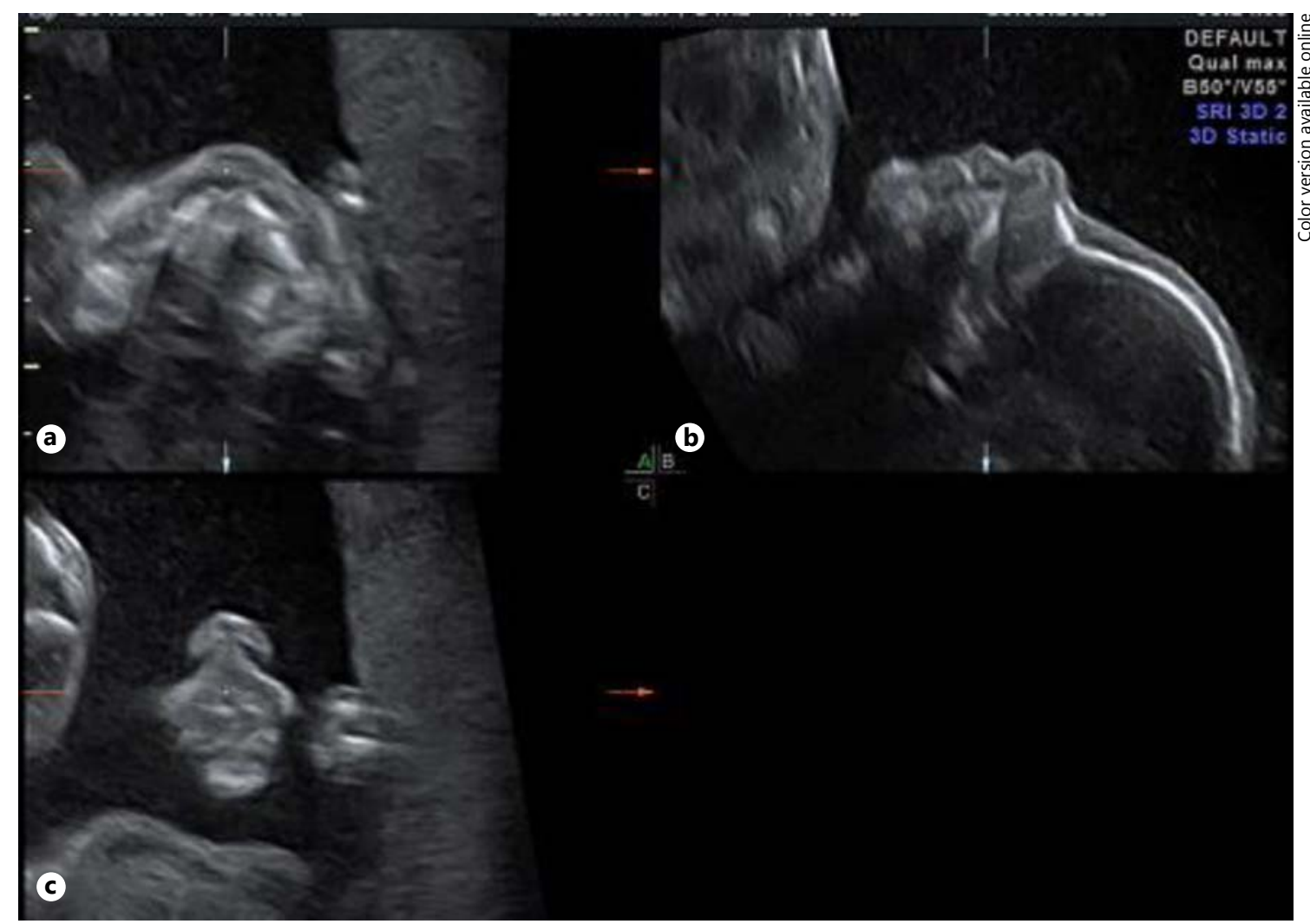

Fig. 2. Image of the reconstructed three orthogonal planes (a axial, b sagittal, $\mathbf{c}$ coronal).

Table 2. Results of the analysis of the different facial planes in the first and second part of the study

\begin{tabular}{lllllllll}
\hline & $\begin{array}{l}\text { Volume } \\
\text { acquired by }\end{array}$ & $\begin{array}{l}\text { Volume } \\
\text { reconstructed by }\end{array}$ & $\begin{array}{l}\text { Number } \\
\text { of volumes }\end{array}$ & $\begin{array}{l}\text { Analysis of } \\
\text { profile in } \\
\text { sagittal view, } \%\end{array}$ & $\begin{array}{l}\text { Analysis of } \\
\text { upper lip in } \\
\text { coronal view, \% }\end{array}$ & $\begin{array}{l}\text { Analysis of } \\
\text { upper lip in } \\
\text { axial view, \% }\end{array}$ & $\begin{array}{l}\text { Analysis of } \\
\text { maxilla in } \\
\text { axial view, \% }\end{array}$ & $\begin{array}{l}\text { Analysis of all facial } \\
\text { components from } \\
\text { same volume, } \%\end{array}$ \\
\hline Part 1 & MFM & IS & 100 & 92 & 95 & 91 & 84 & 72 \\
& IS & IS & 100 & 92 & 82 & 86 & 86 & 76 \\
\hline Part 2 & MFM & 6 different operators & 60 & 93.1 & 86.7 & 87.2 & 86.9 & 77.2 \\
\hline Total & & 260 & 92.4 & 87.9 & 88 & 85.6 & 74.4 \\
\hline
\end{tabular}

MFM = Maternal-fetal medicine specialist; IS = inexperienced sonographer.

II. Analysis of the Volumes Acquired by an

Ultrasound Operator with Minimal Experience in

3DUS Volume Acquisition

In this component of the study, 100 facial volumes without facial clefts were acquired in sagittal (96\%), axial (3\%) or coronal views (1\%). Analysis of the upper lip was possible in $86 \%$ of cases in the axial plane, the maxilla in
$86 \%$ of cases in the axial plane, the profile in $92 \%$ of cases, and the upper lip in $82 \%$ of cases in the coronal plane (table 2).

The reconstruction of all fetal facial components from the same volume (see above) was possible in $76 \%$ of cases.

There was no statistical difference when comparing the analysis of the three orthogonal plane images ac- 


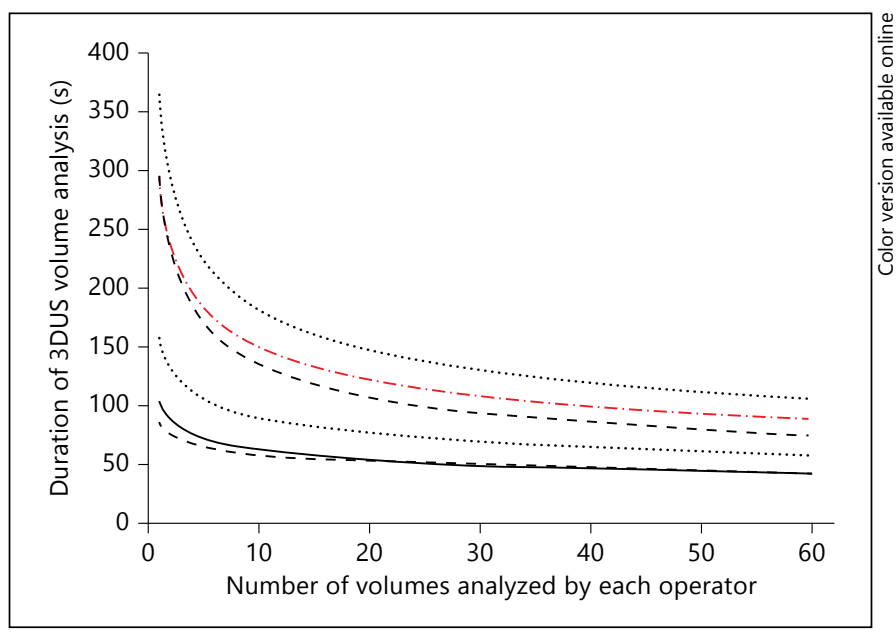

Fig. 3. Learning curve of the 6 investigators (the $\mathrm{x}$-axis represents the number of cases and the y-axis is time in seconds): $-=o b-$ server 1 (maternal-fetal-medicine specialist); - - - = observer 2 (fellow in fetal medicine); - - - observer 3 (fellow in fetal medicine),$\cdots \cdots=$ observer 4 (sonographer with $>8$ years' experience) $\cdot \cdots \cdot=$ observer 5 (sonographer with $>8$ years' experience); $-\cdot-\cdot-=$ observer 6 (resident in gynecology and obstetrics).

quired by a maternal-fetal medicine specialist (part 1 , $72 \%$ ) or by an operator with minimal experience in 3DUS (part 2,76\%, p = 0.629).

\section{Assessment of the Learning Curve Required in Using 3DUS Volumes and the Impact of Image Quality on the Analysis of 3DUS Volumes Acquired by a Maternal-Fetal Medicine Specialist}

Six different ultrasound operators reviewed 60 new volumes, including two isolated cleft lips and five cleft lips which also involved the palate. When considering acquisition, $83.3 \%$ of all volumes were obtained in the sagittal view, $10 \%$ in the axial view and $6.7 \%$ in the coronal view.

The learning curve to reconstruct and analyze three orthogonal plane images was assessed by the time needed to reconstruct adequate planes. A significant improvement in the time needed to reconstruct adequate images was observed between the first 30 and the last 30 volumes $(\mathrm{p}<0.0001)$ for the 6 investigators (fig. 3$)$. The mean improvement was $2 \mathrm{~s}$ per volume up to the 30 th case. For the last 30 volumes, no improvement was observed. The mean time for the first 30 volumes was $125 \mathrm{~s}$ and for the last 30 volumes was $81 \mathrm{~s}$.

Out of the seven cleft lips (total of 42 volumes of cleft lips were analyzed), only 1 of the 6 operators missed the diagnosis of one cleft lip (2.4\%).

3DUS Fetal Face Assessment by

Inexperienced Sonographers
The analysis of the three orthogonal plane images allowed the visualization of the upper lip in $87.2 \%$ of cases in the axial plane, of the maxilla in the axial plane in $86.9 \%$ of cases, of the fetal profile in $93.1 \%$ of cases, and of the upper lip in the coronal plane in $86.7 \%$ of cases (table 2).

\section{Discussion}

Ultrasound examination of the fetal face is routinely performed as part of the morphologic examination to screen for facial anomalies. However, antenatal detection rates of cleft lips with or without cleft palates remain low in the general population $[1,9]$. Difficulty with assessment of the fetal face is multifactorial. Maternal body mass index, operator skill, fetal position, and the amount of amniotic fluid need to be considered. The examiner's technical ability in 2DUS impacts the detection rate of congenital anomalies. With 3DUS, a single-volume acquisition can be used to reconstruct complex $3 \mathrm{D}$ anatomy. We acknowledge that when a $2 \mathrm{D}$ image is not obtained, a 3D image might also be difficult to obtain. However, several authors reported that trained or specialized sonographers have an increased ability to diagnose cleft lips and their severity using 3DUS and need significantly shorter time periods to interpret findings [10-18]. Despite these benefits, the question of whether or not inexperienced sonographers can obtain similar results compared with experienced operators has never been studied. Currently most ultrasound machines have 3D capabilities. In the present study, we (1) described a standardized protocol to reconstruct the fetal face and (2) demonstrated that this $3 \mathrm{D}$ protocol to obtain three orthogonal plane images of the fetal face can be easily used by inexperienced sonographers.

In this study we proposed a simple and effective protocol, using $\mathrm{Z}$ rotation as described by Abuhamad et al. [8]. These manipulations can be performed on ultrasound equipment at the time of the patient examination or later on a laptop workstation from saved volumes.

In the first part of the study, a resident with minimal experience in 3DUS reconstructed the three orthogonal plane images under the supervision of a maternal-fetal medicine specialist. One series of these volumes was acquired by a maternal-fetal medicine specialist and the other series by an operator with minimal experience in 3 DUS volume acquisition. All facial clefts were successfully identified. As shown in table 2 , fetal facial components can be analyzed, regardless of which orthogonal 
plane is used. The reconstruction of the upper lip in the coronal plane, of the maxilla in the axial plane and the profile in the same volume was possible in $72 \%$ of cases when acquired by a maternal-fetal medicine specialist, and $76 \%$ when acquired by an inexperienced operator. Documenting the acquisition time of the volumes was not performed in this study. These results show that there is no difference in facial analysis when the volumes were acquired by a specialist or an operator with minimal experience in 3DUS. Similar results were obtained by other authors for brain volumes [19] or cardiac volumes [20]. Volumes acquired by nonspecialized operators can thus be sent to specialists for further analysis, since the analysis is independent of the operator's level of experience when acquiring the volume. This could decrease the need for unnecessary referrals to specialized units.

In the second part of this study, in which 6 different operators analyzed the same 60 volumes, operators could successfully identify a normal lip and visualize a cleft lip on 3DUS images in $97.5 \%$ of cases. The learning curve for the manipulation of these 3DUS volumes was 30 cases, irrespective of the operator's level of expertise. However, the time needed to work on each volume is operator-dependent, but not experience-dependent: the slower the operators at the beginning of the study, the better they improved with each volume. In other words, the fastest operators did not improve as much as the slowest ones and the more experienced operators were not always the fastest ones.

The sensitivity for facial clefts detection/identification was $97.5 \%$, and only one cleft lip was missed by a single operator. We acknowledge that all of these volumes were acquired by a maternal-fetal medicine specialist before being manipulated by less experienced operators. With such high detection rate, we might expect better detection rate of facial anomalies, especially when fetal ultrasounds are performed by less experienced sonographers, for which 2DUS analysis might be challenging (Clementi and the EUROSCAN Study Group [1] showed a detection rate in Lausanne county of $50 \%$ for cleft lip compared to the $97.5 \%$ detection rate in our study). This promising result should be verified in a routine setting where 2DUS and 3DUS are compared in terms of detection rates of fetal cleft lips and time to obtain the appropriate images.

The high rate of visualization of facial clefts in this study (100\% for the first part and $97.5 \%$ for the second part with a $2.5 \%$ false negative rate and no false positive) could be explained by the fact that the operators were in- cluded in a study concerning fetal face anatomy. Even if the sonographers were unaware of the presence and number of clefts, they were more likely to pay close attention to these anomalies. Finally, retrospective analysis of stored 3DUS volumes might induce potential selection bias. One could argue that stored 3DUS volumes of the fetal face acquired by a maternal-fetal medicine specialist were those with the best visualization. However, similar high performance was obtained when volumes were acquired consecutively by an operator with minimal 3DUS experience. Consecutive acquisition prevented selection bias of the best volumes. After analyzing more than 200 3DUS volumes, we acknowledge that the resident could not be considered inexperienced anymore, since we demonstrated a learning curve of 30 volumes. His capacity to detect cleft lip was thus certainly improved by his participation in the study.

Since we demonstrated that inexperienced sonographers can easily acquire and use 3D volumes of the fetal face as long as they follow a standardized protocol, further studies are now needed to translate 3DUS into routine antenatal care and measure benefits in term of detection rates of facial clefts. In the present study, ability to reconstruct the three orthogonal plane images was assessed. We are currently performing a new study where a standardized acquisition protocol is being evaluated between experienced and inexperienced sonographers.

In conclusion, the present study suggests a high value of 3DUS in the examination of fetal faces, which can be efficiently performed during a routine anatomy scan. Moreover, it confirms the value, performance, learning curve and feasibility of post-processing of acquired volumes for the detection of facial clefts. Indeed, 3DUS for the analysis of the fetal face is reliable and easy to perform, even by inexperienced sonographers. Furthermore, the time required to obtain adequate planes of the face is short enough to be integrated into a routine anatomy ultrasound. 3DUS surfacing allows visualization of the anomaly and a better understanding of the normal and pathologic findings. This can improve understanding and acceptance for the parents [21]. The medical community should be encouraged to incorporate $3 \mathrm{D}$ examinations in their routine imaging for prenatal care with the goal to decrease the common pitfalls in screening for facial clefts and to improve the prenatal detection rate of fetal malformations. 


\section{References}

1 Clementi M, Tenconi R, Bianchi F, Stoll C, and the EUROSCAN Study Group: Evaluation of prenatal diagnosis of cleft lip with or without cleft palate by ultrasound: experience from 20 European registries. Prenat Diagn 2000;20:870-875.

2 Garne E, Loane M, Dolk H, de Vigan C, Scarano G, Tucker D, Stoll C, Gener B, Pierini A, Nelen V, Rösch C, Gillerot Y, Feijoo M, Tincheva R, Queisser-Luft A, Addor M-C, Mosquera C, Gatt M, Barisic I: Prenatal diagnosis of severe structural congenital malformations in Europe. Ultrasound Obstet Gynecol 2005;25: 6-11.

3 EUROCAT Working Group: Appendix 7 and Appendix 8 in Report 8: Surveillance of Congenital Anomalies in Europe 1980-1999. Newtownabbey, University of Ulster, 2002.

4 McGahan MC, Ramos GA, Landry C, Wolfson T, Sowell BB, D'Agostini D, Patino C, Nelson TR, Pretorius DH: Multislice display of the fetal face using 3-dimensional ultrasonography. J Ultrasound Med 2008;27:15731581.

5 Calzolari E, Pierini A, Astolfi G, Bianchi F, Neville AJ, Rivieri F, and the EUROCAT Working Group: Associated anomalies in multimalformed infants with cleft lip and palate: an epidemiologic study of nearly 6 million births in 23 EUROCAT registries. Am J Med Genet Part A 2007;143:528-537.

6 Crane JP, LeFevre ML, Winborn RC, Evans JK, Ewigman BG, Brain RP, Frigoletto FD, McNellis D: A randomized trial of prenatal ultrasonographic screening: impact on the detection, management, and outcome of anomalous fetuses. The RADIUS Study Group. Am J Obstet Gynecol 1994;171:392399.
7 Rey-Bellet C, Hohlfeld J: Prenatal diagnosis of facial clefts: evaluation of a specialised counselling. Swiss Med Wkly 2004;134:640-644.

8 Abuhamad AZ, Singleton S, Zhao Y, Bocca S: The $Z$ technique: an easy approach to the display of the midcoronal plane of the uterus in volume sonography. J Ultrasound Med 2006; 25:607-612.

9 Offerdal K, Jebens N, Syvertsen T, Blaas HG, Johansen OJ, Eik-Nes SH: Prenatal ultrasound detection of facial clefts: a prospective study of 49,314 deliveries in a non-selected population in Norway. Ultrasound Obstet Gynecol 2008;31:639-646.

10 Pretorius DH, House M, Nelson TR, Holenbach KA: Evaluation of normal and abnormal lips in foetuses: comparison between threeand two-dimensional sonography. AJR Am J Roentgenol 1995;165:1233-1237.

11 Merz E, Weber G, Bahlmann F, Miric-Tesanic $D$ : Application of transvaginal and abdominal three-dimensional ultrasound for the detection or exclusion of malformations of the fetal face. Ultrasound Obstet Gynecol 1997;9:237-243.

12 Chen ML, Chang $\mathrm{CH}$, Yu CH, Cheng YC, Chang FM: Prenatal diagnosis of cleft palate by three-dimensional ultrasound. Ultrasound Med Biol 2001;27:1017-1023.

13 Ulm MR, Kratochwil A, Ulm B, Lee A, Bettlheim D, Bernascheck G: Three-dimensional ultrasonographic imaging of fetal tooth buds for characterization of facial clefts. Early Hum Dev 1999;55:67-75.

14 Michailidis GD, Economides DL, Schid RL: The role of three-dimensional ultrasound in obstetrics. Curr Opin Obstet Gynecol 2001;13: 207-214.
15 Ramos GA, Ylagan MV, Romine LE, D’Agostini DA, Pretorius DH: Diagnostic evaluation of the fetal face using 3-dimensional ultrasound. Ultrasound Q 2008;24:215-223.

16 Rotten D, Levaillant JM: Two- and three-dimensional sonographic assessment of the fetal face. 2. Analysis of cleft lip, alveolus and palate. Ultrasound Obstet Gynecol 2004;24:402-411.

17 Benacerraf BR, Shipp TD, Bromley B: Threedimensional US of the fetus: volume imaging. Radiology 2006;238:988-996.

18 Tonni G, Centini G, Rosignoli L: Prenatal screening for fetal face and clefting in a prospective study on low-risk population: can 3 and 4-dimensional ultrasound enhance visualization and detection rate? Oral Surg Oral Med Oral Pathol Oral Radiol Endod 2005; 100:420-426.

19 Rizzo G, Pietrolucci ME, Capece G, Cimmino E, Colosi E, Ferrentino S, Sica C, Di Meglio A, Arduini D: Satisfactory rate of post-processing visualization of fetal cerebral axial, sagittal, and coronal planes from three-dimensional volumes acquired in routine second-trimester ultrasound practice by sonographers of peripheral centers. J Matern Fetal Neonatal Med 2011; 24:1071-1076.

20 Rizzo G, Capponi A, Pietrolucci ME, Capece G, Cimmino E, Colosi E, Ferrentino S, Sica C, Di Meglio A, Arduini D: Satisfactory rate of postprocessing visualization of standard fetal cardiac views from 4-dimensional cardiac volumes acquired during routine ultrasound practice by experienced sonographers in peripheral centers. J Ultrasound Med 2011;30:93-99.

21 Escalon J, Huissoud C, Bisch C, et al: Parental impact of 3D/4D ultrasonography in fetal cleft lip and palate. Gynecol Obstet Fertil 2010;38: 101-104.
3DUS Fetal Face Assessment by Inexperienced Sonographers
Fetal Diagn Ther

DOI: $10.1159 / 000351135$ 